\title{
A Modified Walk Recognition System for Human Identification Based on Uncertainty Eigen Gait
}

\author{
Saad M. Darwish, Adel A. El-Zoghabi, and Oday A. Hassen
}

\begin{abstract}
Gait based recognition is one of the emerging new biometric technology for human identification, surveillance and other security applications. Gait is a potential behavioral feature to identify humans at a distance based on their motion. The use of new methods for handling inaccurate information about gait features is of fundamental important. This paper deals with the design of an intelligent gait recognition system using interval type-2 fuzzy $K$-nearest neighbor (IT2FKNN) for diminishing the effect of uncertainty formed by variations in energy deviation image (EDI). The proposed system is built on top of the well-known principal component analysis (PCA) method that is utilized to remove correlation between the features and also to reduce its dimensionality. Our system employs IT2FKNN to compute fuzzy within and in-between class scatter matrices of PCA to refine classification results. This employment makes the system able to distinguish between normal, abnormal and suspicious walk of a person so that an alarming action may be taken well in time. Interval type-2 fuzzy set is involved to extend the membership values of each gait signatures by using several initial $K$ in order to handle and manage uncertainty that exist in choosing initial $K$. The result of the experiments conducted on gait database show that the proposed gait recognition approach can obtain encouraging accurate recognition rate.
\end{abstract}

Index Terms-Biometric, gait recognition, interval type-2 fuzzy KNN, PCA.

\section{INTRODUCTION}

Gait recognition is a relatively new biometric technology which aims to identify people at a distance by the way they walk (i.e. manner of moving on foot) [1]. Human gait recognition works from the observation that an individual's walking style is unique and can be used for human identification. Gait has its unique advantages for surveillance and monitoring applications in comparison with other biometrics such as iris, fingerprint, and face and so on. Firstly, gait data collection is none contact and unobtrusive (i.e. no human interference is required). Secondly, gait is perceivable at a distance (i.e. it can work well even though the camera situated at large distance). Finally, it is noninvasive and available at low resolution, where other biometrics technologies may be invalid because of insufficient pixels to identify the human subjects [2].

There are several factors that may negatively influence the

Manuscript received March 17, 2014; revised May 19, 2014.

Saad M. Darwish and Adel A. El Zoghabi are with the Department of Information Technology, Institute of Graduate Studies and Research, Alexandria University, 163 Horreya Avenue, El Shatby 21526, P.O. Box 832, Alexandria, Egypt, (tel.: +203)4295007; e-mail: Saad.darwish@alex-igsr.edu.eg, zoghabi@gmail.com).

Oday A. Hassen is with the Department of Mathematics, College of Science, University of Mustansiriya, Iraq (e-mail: oday_ady@yahoo.com). accuracy of biometric gait identification system, which can group into two classes (not necessarily disjoint) [1], [2]: (1) External factors that mostly impose challenges to the recognition approach. For example, viewing angles (e.g. frontal view, side view), lighting conditions (e.g. day/night), outdoor/indoor environments (e.g. sunny, rainy days), clothes (e.g. skirts), walking surface conditions (e.g. hard/soft, dry/wet grass/concrete, level/stairs, etc.), shoe types (e.g. mountain boots, sandals), and object carrying (e.g. backpack, briefcase). (2) Internal factors that cause changes of the natural gait due to sickness (e.g. foot injury, lower limb disorder, Parkinson disease etc.) or other physiological changes in body due to aging, drunkenness, pregnancy, gaining or losing weight. Furthermore, gait recognition is directly linked with quality of segmentation of the walking person. In general, extracting a human silhouette from a video sequence is a challenging task specially when he/she is in contact with some object and background is dynamic i.e. consisting of traffic, running fountains, movement of tree leaves etc.

The procedure of gait recognition includes subject segmentation (extract foreground subjects from video sequences), feature extraction (extract relevant gait features from segmented silhouette sequences), and classification (classify subjects using extracted gait features) [1], [2]. Gait recognition approaches employ both static and dynamic features for recognition [3]. Static features of body are above the waist i.e. head, neck and shoulder etc. Dynamic feature parts are below the waist i.e. foot, legs etc. It is concluded that dynamic parts of the body contains rich information about the gait. Most of the exiting methods adopt both the features and some method adopt dynamic feature only [3], [4].

Gait recognition approaches can be broadly categorized based on gait features into [1], [4]: (1) model-based approaches, where human body structure is explicitly modeled. These approaches are scale, view invariant and require good quality video sequences. In these methods, parameters used as features are the height, the distance between head and pelvis, the maximum distance between pelvis and feet and the distance between feet. These approaches tend to be difficult to implement, as they usually requires some mapping from two to three dimensions. (2) model-free approaches, where gait is treated as a sequence of holistic binary patterns (silhouettes). These methods operate directly on binary silhouettes without assuming any specific model for the walking human. The contour of the silhouette is the most reasonable feature in this method. From other point of view, gait recognition system can be classified depending on the sensors used into three groups namely; motion vision, wearable sensor and floor sensor based. The motion vision 
can be divided into two groups namely; appearance based methods and model based methods. The appearance based method can be also subdivided in two types; state space methods that represent motion as a sequence of poses, and spatio-temporal methods that map the distribution of the motion through space and time. For more details regarding the characteristics of these categories, reader can refer to [2], [3].

Fuzzy logic has emerged as essential methodology for dealing with uncertainty of number environment conditions including viewing angles, lighting conditions, etc. that is always involved in gait recognition application and this a common problem in pattern recognition. Fuzzy logic is used for modeling human thinking and perception [5]. In place of using crisp set (theory of binary propositions), fuzzy systems motive with fuzzy set of multi-values. Fuzzy $k$-nearest neighbor is based on that the pattern set is extended to fuzzy set to assigns class membership to a pattern rather than assigning the pattern to a particular class [6]. For this, it is necessary to select an appropriate initial $K$ for the initialization process. Unfortunately, it is difficult to determine which $K$ is most desirable for the given pattern set. This suggests that uncertainty is present in the selection of initial $K$. Recently, interval type-2 fuzzy $K$-NN algorithm that is an extension of the fuzzy $K$-NN algorithm was proposed [7] In this case, the membership values for each pattern were extended as interval type-2 fuzzy memberships by using several initial $K$. As a result, this method can handle and manage uncertainty that exist in choosing initial $K$.

We open a new category of solution in this paper: type- 2 fuzzy Eigen gait model. We try to enhance the gait recognition rate by utilizing spatio-temporal motion based system that uses principal component analysis (PCA) as data representation to project gait signatures from a high-dimension vector space to some low dimensional space while retaining as much variation as possible in the data set. We apply fuzzy based statistics on PCA by means of employing IT2FKNN in the decision analysis stage as a fuzzy linear classifier. IT2FKNN helps us to find the optimal classification -driven projections of gait patterns that could establish a high degree of similarity between samples of the same gait's class and a high degree of dissimilarity between samples of many gait' classes. In general, type- 2 fuzzy logic systems have been shown to be very well suited to dealing with the large amounts of uncertainties present in the majority of real world applications [8].

The rest of the paper is organized as follows: Section II describes the state-of-the-art gait recognition systems. Section III introduces how to extract the gait signature and presents the pattern classification. Experimental results are reported in Section IV and conclusions are drawn in Section V.

\section{LITERATURE SURVEY}

A good number of algorithms are reported in the literature for model- free gait recognition, which has been develop using technique from mathematics, statistics and computer science [9]-[11]. Model-free approaches are insensitive to the quality of silhouettes and have the advantage of low computational costs comparing to model-based approaches.
However, they are usually not robust to viewpoints and scale. Most of these algorithms use the silhouettes themselves as features, which are scaled and aligned before used. In [2] some scholars proposed the motion-energy image (MEI) and motion-history image (MHI) to convert the temporal sequence of silhouettes to a 2D signal template. Some researchers employed the idea of MEI and they suggested the Gait Energy Image (GEI) for individual recognition. GEI converts the spatial-temporal information during one walking cycle into a single $2 \mathrm{D}$ gait template, which avoids matching features in temporal sequences. GEI is comparatively robust to noise by averaging images of a gait cycle. However, it loses the dynamical variation between successive frames [10].

To address the problem of silhouette incompleteness, the authors in [12] proposed the frame difference energy image (FDEI) based on GEI and Gait History Image (GHI) that retains temporal information as well as spatial information. Other authors applied the wavelet decomposition of GEI to realize gait recognition. The established gait sequences are robust to the covariates of holding a ball and loading packages. Moreover, the silhouette itself as features may be more suitable for low quality and low resolution data [4], [13]. Later, some academics combined the entire silhouette and the width of outer contour silhouette as gait features. Some other algorithms pay attention to analyzing the whole shape of silhouettes. For instance, some investigators performed radon transform on the binary silhouettes to get a template from gait sequences. Linear discriminate analysis (LDA) and subspace projection are used to extract radon template coefficients to construct the feature vector [2].

Regarding gait classification, direct gait classification methods do not pay attention to the temporal information of gait sequences. They are based on the single representation or key frames extracted from a sequence of gait frames. For instance, $K$-nearest neighbor classifier decides the class of test feature according to the number of the $k$ closest training examples [3]. Some authors used various discriminative classifiers. For example, support vector machine (SVM) is used in [14] to classify features extracted from GEI. SVM is considered as a generalized linear classifier and is a supervised learning method.

In the literature, PCA and LDA are traditional but widely used feature reduction methods. For example, some examiners in [15] applied PCA to time-varying distance signals derived from silhouette images sequence to reduce the dimensionality of feature space. However, PCA-based methods only preserve those features which contribute most to variance, which may be not optimal for classification. More recently, some algorithms perform PCA and LDA together to improve the topological structure and reduce the dimensionality of the feature space. Another related work in [16] that combined PCA and fuzzy logic to process GEI. A Short summary of the existing literature on gait recognition approaches can be found in [1]-[4].

In this paper, we have endeavored to enhance the existing fuzzy-based gait recognition algorithms and introduce a type-2 fuzzy gait recognition system with the objective of boosting individual recognition rate by type-2 fuzzy KNN (T2FKNN) technique. The proposed system represents 
subjects based on energy deviation image (EDI), which is a good complement of the GEI that ignores some gait motion information. Then, applying type-2 fuzzy set in nearest neighbor classifier to identify the human. Through PCA and IT2FKNN, gait feature can keep the information of original data and reduce the dimension of the feature effectively. Furthermore, classification results can be refined so that they could affect the within-class and between-class scatter matrices of human gaits through managing the uncertainty for the selected initial $K$.

\section{ALGORITHM DESCRIPTION}

Fig. 1 shows the gait recognition system used in this paper, which comes with better classification performance. The training and recognition phases of our approach make use of the following steps: Background Subtraction, periodicity detection, feature extraction and reduction, and IT2FKNN recognition. Firstly, the original gait sequence is preprocessed and EDI' features are obtained. Secondly, we extract the eigenvalues and associated eigenvectors. Then the eigenvectors are projected into lower-dimensional Eigen space. After that T2 fuzzy nearest neighbor (NN) is adopted as an extra layer to achieve a good discriminating ability compared to PCA under server variation in viewing angles, and lighting conditions. Finally, Euclidian distance is used to classify the gait feature. The following subsections describe the processing steps in more details.

\section{A. Background Subtraction and Silhouette Decomposition}

In order to study the cyclic human action, we need to segment the objects from the background. Gait signature extraction is the key task in human gait recognition. It must be reasonably robust to the varying conditions and should yield good discrimination across individuals. Intuitively, the silhouette appears to be a good feature to utilize since it captures the motion of most of the body parts and also encodes structural as well as transitional information [10]. Particularly, Silhouette is independent of the clothing, illumination and textures, etc.

The aim is to produce binary images of the person from the video sequence by using certain method that comprises three important stages, which are background generation, silhouette extraction and morphological operation [9], [17]. Background generation is accomplished by robustly estimating the statistics (mean) of the background and segmenting any pixels that do not fit the statistics. Median filtering along the temporal direction is performed for each pixel to estimate the mean of its background value. Because of unstable light sources, small blobs always appear. Here, we make use of spatial median filtering in each segmented image to remove the small blobs.

In formal, the spatial median of a set of points $p_{1}, \ldots \ldots p_{N}$ is calculated as follows [18]: (1) for each vector $X$ compute $S_{\text {depth }}$ that is a set of the sum of the spatial depths from $X$ to every other Vector, (2) find the maximum spatial depth of this set, $S_{\max }$, (3) $S_{\max }$ is the spatial median of the set of points. In general, spatial median filter is an unbiased smoothing algorithm and will replace every point that is not the maximum spatial depth among its set of mask neighbours.

$$
S_{\text {depth }}\left(X, p_{1}, \ldots \ldots p_{N}\right)=1-\frac{1}{N-1}\left|\sum_{i=1}^{N} \frac{X-p_{i}}{\left|X-p_{i}\right|}\right|
$$

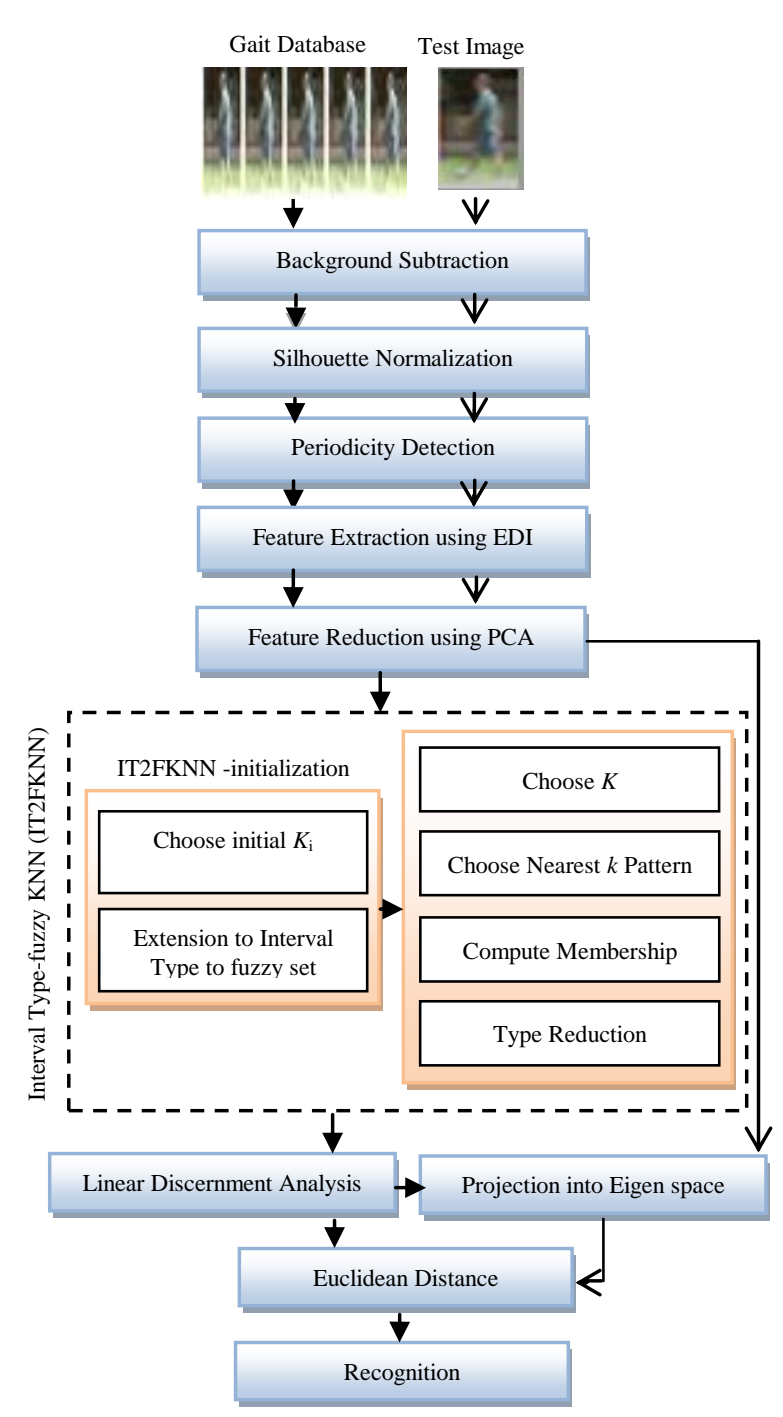

Fig. 1. Block diagram of the proposed type-2 fuzzy gait recognition system.

The silhouette is usually extracted by finding the difference between the background and current image. The difference image must be converted into a binary image by applying a suitable threshold value. In many cases, there are inevitably holes inside moving subject and other anomalies in the detected gaits caused by body portion lost that has a greater effect on recognition than other errors like shadows and spurious pixels. Mathematical morphological operations, such as erosion and dilation, are widely used to remove spurious pixels and fill small holes inside the extracted silhouettes. Fig. 2 shows example of silhouette extraction.

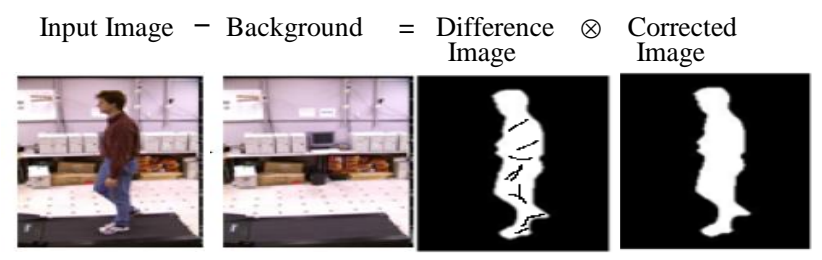

Fig. 2. Illustration of background subtraction. 


\section{B. Silhouette Normalization}

Normalization is required as a pre-processing step in every pattern recognition system. The objective is to reduce the sensitivity of the localization, orientation and scale estimation with respect to silhouette deformations [19]. To eliminate the size difference caused by the varying distance between the subject and camera, the silhouettes are usually height scaled and centered. We have to normalize the size of binary silhouettes to a fixed width $\mathrm{X}$ height bounding rectangle. It will then be possible to center and align in a similar manner different silhouettes that belong to a same object or class of objects. Fig. 3 shows example of silhouette normalization.

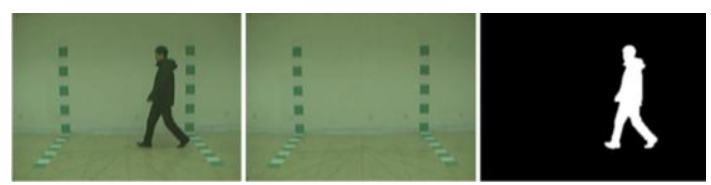

(a)

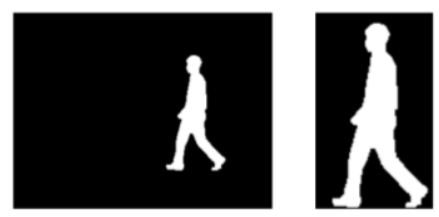

(b)

Fig. 3. (a) Extraction foreground image (b) Normalized and centralized image.

\section{Periodicity Detection}

The gait of a person is a periodic activity with each gait cycle covering two strides - the left foot forward and right foot forward strides. Each stride spans the double-support stance to the legs-together stance and back to the double-support stance as shown in Fig. 4. We utilize the method proposed in [10], [12] to estimate the gait period of a walking sequence that is tolerant to low quality silhouette images. A silhouette's swing distance $s w$ is obtained as follows:

$$
s w=\sum_{y=y_{b}}^{y_{t}-\frac{\left(y_{t}-y_{c}\right)}{2}} \sum_{x=x_{t}}^{x_{r}}\left|\left(x-x_{c}\right) \times \frac{I(x, y)}{255}\right|
$$

where $\left(x_{c}, y_{c}\right)$ is the centroid of the silhouette, $x_{t}$ and $x_{r}$ denote the horizontal positions of the left-most and the right-most boundary pixels of the silhouette respectively, $x_{b}$ and $x_{t}$ denote the vertical positions of the bottom-most and the top-most boundary pixels of the silhouette respectively, and $I(x, y)$ denotes the intensity of the pixel $(x, y)$. The variation of swing distance shows the periodicity of gait.

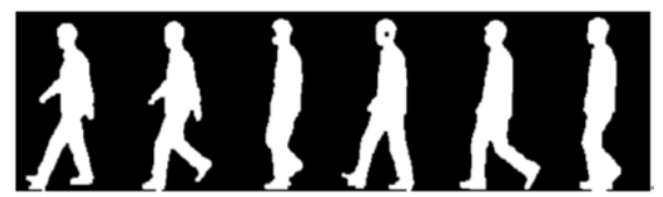

Fig. 4. Silhouette image of one circle.

The frame with local maximum $s w$ is the frame in double support stance, and the frame with local minimum $s w$ is the frame in legs-together stance. Let $d_{i}$ be the $i^{\text {th }}$ difference in the sorted list that contains the difference of frame number between every two adjacent odd maximum frame, and let $C$ be the number of the differences in the sorted list, i.e. the number of gait cycles in the video sequence. The gait period of current subject can be estimated as follows [10]:

$$
d_{h}=\frac{2}{c} \times \sum_{p i=\frac{c}{4}}^{\frac{3 c}{4}-1} d_{i}
$$

\section{Feature Extraction Using EDI}

The main step of gait recognition is to extract the appropriate salient features that effectively describe the motional characteristics of the parts of body. Features extracted are of two types. First, those which detach the foreground and generate silhouettes as illustrated above. Second, those which use to identify a person. These feature variables should be invariants under environmental conditions like viewing angles.

This work employed energy deviation image (EDI) as gait feature vector to represent subjects [20]. EDI is a good complement of the gait energy image (GEI) that has been reported as a good feature robust to silhouette errors and image noise but ignores motion gait features. EDI captures not only static but also motion characteristic of gait. On the basis of calculated gait period, deviation from GEI at the $i^{\text {th }}$ frame in the $c^{\text {th }}$ gait cycle is expressed as [12]:

$$
\text { dif } f_{h c}=\left|f_{h c}(x, y, i)-g_{h}(x, y)\right|
$$

where $f_{h c}(x, y, i)$ is the original silhouette image at the frame in the $c^{\text {th }}$ gait cycle and $g_{h}(x, y)$ is the gait energy image of the subject $h$, which is the average silhouette over one gait cycle.

$$
g_{h}(x, y)=\frac{1}{m} \sum_{i=1}^{m} f_{h i}(x, y, i)
$$

$m$ is the number of frames contained in current gait cycle and $f_{h i}(x, y, i)$ is the $i^{\text {th }}$ silhouette image sequence for the subject $h$. From the calculated dif $f_{h i}$, EDI of the $C^{\text {th }}$ gait cycle is computed recursively by:

$$
\operatorname{devf} f_{h c}(x, y, g)=\left\{\begin{array}{c}
\max \left(\operatorname{dev} f_{h c}(x, y, g-1)-\frac{255}{d_{h}}, 0\right) \\
\text { if } \operatorname{dif} f_{h c}(x, y, g)=0 \\
\left.\max \left(\operatorname{dev} f_{h c}(x, y, g-1), \operatorname{dif} f_{h c}(x, y, g)\right)\right) \\
\text { else }
\end{array}\right.
$$

where value of $g$ ranges from 1 to $\mathrm{s}$, that represents the sequence number of current gait silhouette in the $c^{\text {th }}$ gait cycle. So, the result of the equation at the $s^{\text {th }}$ step is the energy deviation image of $c^{\text {th }}$ gait cycle. Finally, the energy deviation image is calculated as:

$$
\operatorname{devf}_{h}(x, y, s)=\frac{1}{c_{h}} \sum_{c=1}^{c_{h}} \operatorname{dev} f_{h c}(x, y, s)
$$

here, $c_{h}$ is the number of gait cycles in the current walking sequence of the subject $h$. Fig. 2(a) shows the EDI 
corresponding to 4 gait cycles, and Fig. 5(b) demonstrates the final $E D I$ as the average of all EDI mentioned in Fig. 5(a)

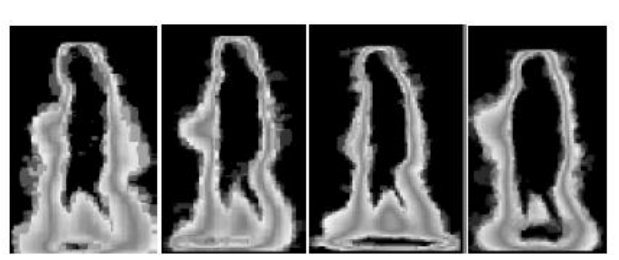

(a)

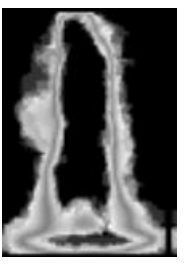

(b)
Fig. 5. (a) Energy deviation image of gait cycle of a subject. (b) Final energy deviation image.

\section{E. Feature Reduction Using PCA (Eigen Gait)}

Since, the gait feature space obtained from EDI is very high dimensional, so we transform it into low dimensional space by $P C A$ (Eigen gait). Thus, very few components are required to represent the subject while retaining as much variation as possible in the data set [15]. PCA practically transforms the original data matrix into product of two matrices, one of which contains the information about the samples and the other about the variables [21]. The $D$ matrix contains the scores of the $h$ subjects on $Q$ principal components. The $V$ matrix is a square matrix and contains the loadings of the original variables on the principal components. Express the corresponding covariance matrix $M$ in the standard manner as:

$$
\begin{gathered}
M=E[B \otimes B]=E\left[B \cdot B^{*}\right]=\frac{1}{N_{h}} \sum B \cdot B^{*} \\
B=\operatorname{devf}_{h}-\left(U_{M \times 1} \times O_{1 \times N}\right) \\
U=\frac{1}{N_{h}} \sum_{h=1}^{N_{h}} \operatorname{dev} f_{h}(x, y, s)
\end{gathered}
$$

where $B$ is the expected value, $N_{h}$ is the number of subject, and $\otimes$ is the outer product. The computed matrix $V$ of eigenvectors which is diagonalizable the covariance matrix $M$ is defined as $V^{-1} C V=D$, in which $D$ is the diagonal matrix of eigen values of $M$.

\section{F. Interval Type-2 Fuzzy K-Nearest Neighbor Classifier}

To improve the performance of the recognition, the system uses interval type-2 fuzzy K-NN (IT2FKNN) to refinement of recognition results so that they could affect the within-class and between-class gaits' features vector. In this stage the membership values for each Eigen gait extracted from the previous stage are extended as interval type-2 fuzzy memberships by assigning uncertainty to the type-1 memberships. By doing so, the classification result obtained by the interval type- 2 fuzzy K-NN is found to be more reasonable than that of the crisp and type-1 fuzzy K-NN [7].

In formal, given $X_{i}$ as a set of Eigen gait obtained from the previous stage, IT2FKNN partition of these vectors specifies the degrees of membership of each vector to the gait classes or subjects. Let the partition matrix denoted by $u=\left[u_{i}\right], i=1,2, \ldots \ldots N_{h}$, the assigned membership of the gait pattern $X$ is computed as:

$$
u_{i}(X)=\frac{\sum_{j=1}^{K} \tilde{u}_{i j}\left(1 /\left\|x-x_{j}\right\|^{2 /(m-1)}\right)}{\sum_{j=1}^{k}\left(1 /\left\|x-x_{j}\right\|^{2 /(m-1)}\right)}
$$

here $u_{i}$ represents the interval type- 2 fuzzy membership of for class $i, \quad \tilde{u}_{i j}$ denotes initial interval type-2 membership of $x_{j}$ which is the $i^{\text {th }}$ nearest neighbor to $x$ in class $i$, and $m$ symbolizes degree of fuzzification. In this case, several initial $K$ values for $K N N$ algorithm are used to manage and control the uncertainty for selecting an appropriate initial $k$ for the initialization process.

This step consists of 2 stages. In the first stage, we perform Interval Type-2 Fuzzy K-Initialization to extend pattern set to interval type-2 fuzzy sets. This is used for determining the elements of primary memberships on IT2FS. In the next stage, we perform interval type-2 fuzzy K-NN with interval type-2 fuzzy set. In this part, we assign interval type-2 fuzzy membership for a given pattern using the union operation. Finally, when we classify pattern by membership grade, we perform type- reduction and defuzzification. In this process, we can reduce redundant primary membership values. However, since we use an IT2FS, we not consider secondary grade, that is always 1 . Herein, a specific relation between initial $\mathrm{K}$ and primary membership is as:

$$
1 \leq \bigcup_{r=1}^{n} u\left(j, k_{r}, x_{i}\right) \leq n \quad, k_{r}=\left\{k_{1}, k_{2}, \ldots . k_{n}\right\}
$$

where $u\left(j, k_{r}, x_{i}\right)$ characterizes the primary membership of the $i^{\text {th }}$ gait pattern in class or individual $j$ when initial $K$ is selected as $k_{r}$. Furthermore, the primary memberships of each gait can be represented as:

$$
u_{i}\left(X_{N}\right)=u_{1_{i N}}+\ldots \ldots+u_{R_{i N}}=\sum_{r=1}^{R_{i N}} u_{i N}
$$

$R_{i N}$ denotes the number of primary membership for $X_{N}$ with the number of primary memberships for $K$ nearest neighbor patterns. To extend the primary membership for given pattern to an IT2FS, Eq. (13) turns into:

$$
u_{i}\left(X_{N}\right)=\sum_{r=1}^{R_{i N}} 1.0 / u_{r_{i N}}
$$

for the type reduction process, the type-reduced membership of $x_{N}$ in class $i$ can be expressed as:

$$
u_{i}\left(X_{N}\right)=\frac{\sum_{r=1}^{R_{i N}} f\left(u_{r_{i N}}\right) u_{r_{i N}}}{\sum_{r=1}^{R_{i N}} f\left(u_{r_{i N}}\right)}=\frac{\sum_{r=1}^{R_{i N}} 1.0 \times u_{r_{i N}}}{\sum_{r=1}^{R_{i N}} 1.0}=\frac{\sum_{r=1}^{R_{i N}} u_{r_{i N}}}{N_{h}}
$$

in which $f\left(u_{r_{i N}}\right)$ represents secondary grade. Therefore, it is approximately the average of primary memberships of interval type-2 fuzzy set.

In summary, in the type-1 fuzzy case, only one initial $K$ is selected to assign initial fuzzy memberships to the pattern data. If the selection of $K$ is poor, an undesirable classification rate for the pattern data can be obtained. However, for the interval type-2 fuzzy approach, we need not select only one initial $K$. This is due to the extension of the pattern data into an interval type-2 fuzzy set. For this extension, we use initial $K$ values in an appropriate range. Handling of this uncertainty can decrease the contribution of an undesirable initial $K$ on the classification process for the patterns. Hence, this can provide a more reasonable classification result by managing 
the uncertainty for the selected initial $K$. Reader looking for more details can refer to [5]-[7].

\section{G. Linear Discriminate Analysis}

Taking into account the membership grades $u_{i j}$ obtained from IT2FKNN classifier step, the statistical properties of the patterns (gaits) such as the mean value and scatter covariance matrices are used to find the optimal classification-driven projection of patterns. $u_{i j}$ is incorporated into the definition of the between-class scatter matrix and within-class scatter matrix to get the fuzzy between and within-class scatter matrix as follows [22]:

$$
\begin{gathered}
\tilde{m}_{i}=\frac{\sum_{j=1}^{N_{h}} u_{i j} x_{j}}{\sum_{j=1}^{M} u_{i j}} \\
s_{b}=\sum_{i=1}^{N_{h}} N_{i}\left(\tilde{m}_{i}-\bar{m}\right)\left(\tilde{m}_{i}-\bar{m}\right)^{T} \\
S_{w}=\sum_{i=1}^{N_{h}} \sum_{x_{s \in c_{i}}}\left(x_{s}-\tilde{m}_{i}\right)\left(x_{s}-\tilde{m}_{i}\right)^{T}=\sum_{i=1}^{N_{h}} s_{w i}
\end{gathered}
$$

where $\tilde{m}_{i}$ is the mean vector of class $i, \bar{m}$ stands for the mean of all gait' vectors, $M$ represents the numbers of classes and both between-class fuzzy scatter matrix $S_{b}$ and within-class fuzzy scatter matrix $s_{w}$ incorporate the membership values in their calculations. The optimal interval type 2 fuzzy projection $W_{I T 2 F K N N}$ follows the expression.

Once $s_{b}$ and $s_{w}$ are computed, it is recommended to find the optimal projection axis $W$ so that the total scatter of the projected samples of the training images is maximized. The objective function can be defined as:

$$
W_{\text {IT2FKNN }}=\arg \max _{w} \frac{\left|w^{T} s_{b} w\right|}{\left|w^{T} s_{w} w\right|}
$$

In this case, the feature vector transformed by interval type-2 fuzzy linear discriminate analysis method follows the expression:

$$
\tilde{v}_{i}=w_{I T 2 F K N N}^{T} x_{s}
$$

Finally, recognition is done using Euclidian distance. Euclidian distance between feature vector of input real-time image and feature vector of each training image is calculated using following formula.

$$
E D=\sqrt{\left(\tilde{v}_{i+1}^{1}-\tilde{v}_{i}^{1}\right)^{2}+\left(\tilde{v}_{i+1}^{2}-\tilde{v}_{i}^{2}\right)^{2}}
$$

If the Euclidean distance between frame $A$ and frame $B$ in the training database is smaller than a fixed threshold value T, then frames $A$ and $B$ are proved to be the same subject. The threshold $T$ is the largest Euclidean distance between any two images in the training database, divided by a threshold tuning value $(\zeta)$. The value of the threshold tuning parameter can be used to tune the performance of the system to have either high correct recall with high false acceptance rate for application such as boarder monitoring or high correct rejection rate for unknown persons for application such as access control.

\section{EXPERIMENTAL RESULTS}

Our experiments are carried out on the CMU MoBo gait database as it is freely available [23]. In this database, 25 subjects are trained on the tread mill. Around the tread mill, the six cameras have set to capture the images in six different viewing angles. The provided database has four kinds of walking pattern which are slow, fast, incline and carrying a ball walk. Each subject walking pattern has six kind of view in different angles. Each view captured 340 frames that can be calculated minimum 14 gait cycles and each cycle has generally 18 to 20 frames. One cycle frames are combined and prepared an EDI frame. So one subject got minimum 14 EDI images. For the training and testing, 10 EDI frames selected for training and 4 EDI frames selected for testing to perform the experiment for one subject. Total number of EDI training and testing frames are $13 \times 10=130$ and $13 \times 4=52$ frames respectively. For the unknown subject, one cycle has been selected to make EDI unknown frames.

TABLE I: EXPERIMENTAL DESIGN

\begin{tabular}{cccccccccc}
\hline $\begin{array}{c}\text { Experiment } \\
\text { Label }\end{array}$ & $\mathrm{A}$ & $\mathrm{B}$ & $\mathrm{C}$ & $\mathrm{D}$ & $\mathrm{E}$ & $\mathrm{F}$ & $\mathrm{G}$ & $\mathrm{H}$ & $\mathrm{I}$ \\
\hline $\begin{array}{c}\text { Size of the } \\
\text { Inquiry set } \\
\text { Gallery }\end{array}$ & 120 & 60 & 60 & 120 & 60 & 120 & 120 & 60 & 120 \\
\hline \hline
\end{tabular}

(V-view, H-Shoe, S-Surface, B-Briefcase)

Nine experiments are designed for individual recognition as shown in Table I. In the experiments, the parameter $\zeta$ was set so that the system has equal false accept rate (FAR) and false reject rate (FRR). Our experiments begin with binary silhouette data extracted by applying baseline approach [24]. The number of principle components $Q$ is chosen to facilitate the solution of Eq. 20 such that $Q=2 \mathrm{c}$, where c is the number of classes. The experimental results as well as comparison with other approaches of individual recognition by gait are shown in Table II. In this table, rank 1 and 5 performances mean the percentage of the correct subjects appearing in the first place and first five places of the retrieved rank respectively. Table II compares the recognition performance of baseline algorithm and our proposed method.

It can see that the rank 1 and 5 performances of the proposed method are better than the baseline algorithm on all experiments but slightly worse on $D$ for rank 5 . This shows the inherent representational power of EDI and demonstrates that matching features learned from IT2FKNN classifier achieve better recognition performance than direct matching between individual silhouette frame pairs in the baseline algorithm.

TABLE II: COMPARISON OF RECOGNITION PERFORMANCE (\%)

\begin{tabular}{c|cc|c|c}
\hline \hline & \multicolumn{2}{|c|}{ Rank 1 Performance } & \multicolumn{2}{c}{ Rank 5 Performance } \\
\cline { 2 - 5 } & Baseline & Proposed & Baseline & Proposed \\
\hline A & 73 & 93 & 88 & 98 \\
B & 78 & 92 & 93 & 95 \\
C & 48 & 83 & 78 & 93 \\
D & 32 & 60 & 66 & 80 \\
E & 22 & 65 & 55 & 84 \\
F & 17 & 30 & 42 & 60 \\
G & 61 & 70 & 85 & 92 \\
H & 57 & 63 & 78 & 86 \\
I & 36 & 61 & 62 & 83 \\
\hline \hline
\end{tabular}


Fig. 6 shows the Receiver Operating Characteristic curve (ROC) between FAR and FRR with different threshold values $T$. These curves are quite helpful in selecting correct value of threshold so that FAR and FRR both can be kept minimum. In our case it is $3 \%$. Although the proposed method achieves significantly better results than baseline algorithm, its performance is still not satisfactory in the presence of the large silhouette distortion in probe set contains clothing such as person wearing muffler in the neck or wearing shawl. In all cases the recognition rate is below $50 \%$.

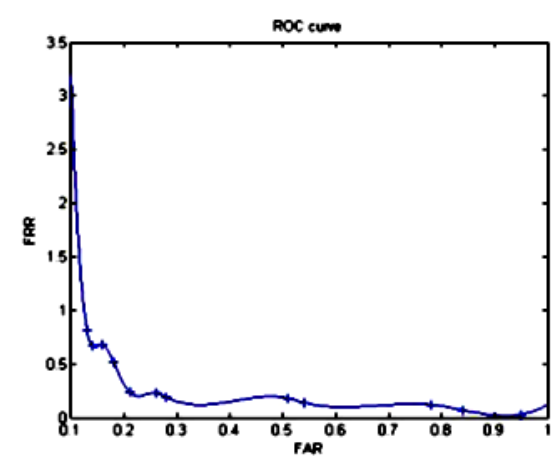

Fig. 6. ROC curves with different Threshold value.

\section{CONCLUSION}

Gait is a prospective behavioral feature and many related studies have demonstrated that it has a rich potential as a biometric for recognition. This paper has proposed an enhanced and effective method for gait-based human recognition by means of employing both of EDI, PCA, and type-2 fuzzy KNN-based discriminate analysis. The proposed motion-based system operates on the silhouettes of a subject, which can be easily acquired through preprocessing methods. EDI is obtained based on the difference between original silhouette image and gait energy image, and utilized to represent a subject. By PCA, the largest eigenvalues and associated eigenvectors are achieved while the components are projected into the lower dimension space. IT2KNN classifier is adopted for diminishing the effect of uncertainty formed by variations in EDI and to identify the human.

The key advantages include low computation cost and unnecessary learning by large data, both of which suggest it to be real-time and efficient for video surveillance. Our approach is robust over variations in backgrounds, walking and running speeds, direction of motion (left or right), and, to some degree, distance to the camera. Moreover, by considering various initial $K$ values for the pattern memberships inside $K N N$, a more reasonable classification result may be obtained. The suggested system requires clear-cut images; pre-processed images could be implemented for intact human silhouette before feature extraction and representation. One limitation common to our approach and most previous approaches is that the motion must be aside parallel.

The results were compared with another published paper and reported that the proposed system gives efficient result on the CMU MoBo gait database. It is clear from our experiments that the assumptions made on dynamic features of human body (i.e. hand and feet) increase gait recognition and the average classification. In the future, we will attempt to enhance the proposed system by combining different view sequences as training data that may provide an effective way to solve the problem of gait view variant. Further future work includes utilizing other complex classification algorithms to achieve higher recognition rate.

\section{REFERENCES}

[1] D. Gafurov, "A survey of biometric gait recognition: Approaches, security and challenges," in Proc. IEEE International Conference on Biometrics: Theory, Applications and Systems, pp.1-12, Norwegian, 2007.

[2] E. B. Jeffrey and J. L. James, "Biometric gait recognition," in Proc. the

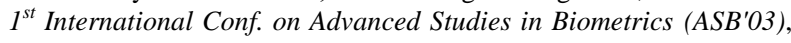
vol. 3161, pp. 19-42, Berlin, 2005.

[3] G. Praveen, K. Rajendra, K. Rohit, and R. Ritesh, "Biometric system based on human gait patterns," International Journal of Machine Learning and Computing, vol. 1, no. 4, pp. 378-387, 2011.

[4] A. Hayder, J. Dargham, A. Chekima, and G. M. Ervin, "Person identification using gait," International Journal of Computer and Electrical Engineering, vol. 3, no. 4, pp. 477-482, 2011.

[5] B. Jyoti and M. K. Gupta, "Gait recognition with fuzzy classification using shoulder body joint," International Journal of Future Computer and Communication, vol. 2, no. 6, pp. 590-594, 2013.

[6] Y. K. Kim and J. H. Han, "Fuzzy K-NN algorithm using modified $k$ selection," in Proc. IEEE International Conf. on Fuzzy Systems, vol. 3 , pp. 1673 - 1680, Japan, 20-24 Mar. 1995.

[7] F. C. Rhee and C. Hwang, "An interval type-2 fuzzy $k$-nearest neighbour," in Proc. International Conf. on Fuzzy Systems, pp. 802-807, USA, 25-28 May 2003.

[8] L. Qilian and J. M. Mendel, "Interval type-2 fuzzy logic system: theory and design," IEEE Trans. on Fuzzy Systems, vol. 8, no. 5, pp. 535-550, October 2000.

[9] A. Hayder, D. Jamal, A. Chekima, and E. G. Mong, "Gait recognition using principle component analysis," in Proc. International Conf. on Machine Vision (ICMV2010), pp. 539-543, Malaysia, 2010.

[10] A. Hayder, D. Jamal, A. Chekima, and E. G. Moung, "Gait recognition using gait energy image," International Journal of Signal Processing, Image Processing and Pattern Recognition, vol. 4, no. 3, pp. 141-152, 2011.

[11] C. Javier, A. Josep, and D. L. Fernando, "Robust normalization of silhouettes for recognition applications," Journal of Pattern Recognition, vol. 25, no. 5, pp. 591-601, 2004.

[12] M. Qinyong, W. Shenkang, N. Dongdong, and Q. Jianfeng, "Gait recognition at a distance based on energy deviation image," in Proc. $1^{\text {st }}$ International Conf. on Bioinformatics and Biomedical Engineering (ICBBE), pp. 621 - 624, China, 2007.

[13] Z. Xue, D. Ming, W. Song, B. Wan, and S. Jin, "Infrared gait recognition based on wavelet transform and support vector machine," Pattern Recognition, vol. 43, issue 8, pp. 2904-2910, Aug. 2010.

[14] L. Du and W. Shao, "Recognizing gait using Haar wavelet and support vector machine," Advances in Intelligent and Soft Computing, vol. 158, pp. 221-227, 2012.

[15] M. Pushparani and D. Sasikala, "A survey of gait recognition approaches using PCA \& ICA," Global Journal of Computer Science and Technology Network, Web \& Technology, vol. 12, no. 10, pp. 7-10, 2012.

[16] G. N. Venkata and S. Jilani, "Fuzzy principal component analysis based gait recognition," International Journal of Computer Science and Information Technologies (IJCSIT), vol. 3, no. 3, pp. 4015 - 4020, 2012.

[17] Q. Ma, S. Wang, D. Nie, and J. Qiu, "Recognizing humans based on gait moment image," in Proc. International Conf. on Software Engineering, Artificial Intelligence, Networking, and Parallel/Distributed Computing, vol. 2, pp. 606-610, China, 2007.

[18] C. James, C. Yixin, and R. Stephen, "A spatial median filter for noise removal in digital images," in Proc. IEEE Southeast Conference, USA, pp. 618-623, 2008.

[19] B. Jyoti and M. K. Gupta, "Gait recognition with geometric characteristic and fuzzy logic," International Journal on Image Processing and Computer Vision, vol. 3, no. 1, pp. 6-11, 2012.

[20] K. Rohit and V. K. Pathak, "Gait recognition based on energy deviation image using fuzzy component analysis," International 
Journal of Innovation Management and Technology, vol. 4, no. 1, 2013, pp. 43-46.

[21] L. L. Li and L. Z. Wang, "Gait recognition using kernel two dimensional principal component analysis," Journal of Computational Information Systems, vol. 9, no. 3, pp. 837-844, 2013.

[22] W. Yang, J. Wang, M. Ren, and J. Yang, "Fuzzy 2-dimensional FLD for face recognition," Journal of Information and Computing Science, vol. 4, no. 3, pp. 233-239, 2009.

[23] G. Ralph and S. Jianbo, The CMU Motion of Body (MOBO) Database, Carnegie Mellon University, 2001

[24] S. Sarkar, P. Phillips, Z. Liu, L. Vega, P. Grother, and K. Bowyer, "The human gait challenge problem: Data sets, performance, and analysis," IEEE Trans. Pattern Analysis and Machine Intelligence, vol. 27, no. 2 pp. 162-177, Feb. 2005.

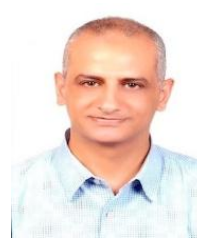

Adel A. El-Zoghabi received his B.Sc. in computer engineering from Alexandria University in 1987, his M.Sc. and Ph.D. in information technology from Alexandria University and Old Dominion University in 1991 \& 1994 respectively. His research and professional interests include intelligent systems and machine learning, inter networking \& routing protocols, and distributed systems. He has published many papers in international journals and conferences worldwide during the past three decades. Currently he is a professor of computer science and IT and the head of Dept. of Information Technology since August 2012.

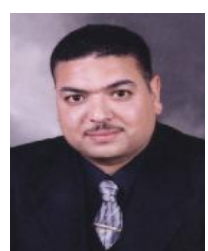

Saad M. Darwish received his Ph.D. degree from the Alexandria University, Egypt. His research and professional interests include image processing, optimization techniques, security technologies, and machine learning. He has published in journals and conferences and severed as TPC of many international conferences. Since Feb. 2012, he has been an associate professor in the Department of Information Technology, Institute of Graduate Studies and Research, Egypt.

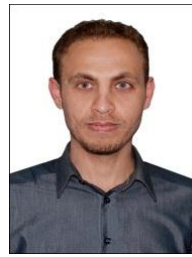

Oday A. Hassen received the B.Sc. degree in mathematics from the Department of Mathematics, University of Mustansiriyah, Iraq in 2004. Currently he is a M.Sc. student in the Department of Information Technology, Institute of Graduate Studies and Research, Alexandria University, Egypt. His research and professional interests include image processing, authentication and security technologies. 